\title{
Assessment of in vivo microstructure alterations in gray matter using DKI in internet gaming addiction
}

Yawen Sun ${ }^{1+}$, Jinhua Sun ${ }^{2 \dagger}$, Yan Zhou ${ }^{1{ }^{*+}}$, Weina Ding ${ }^{1}$, Xue Chen ${ }^{1}$, Zhiguo Zhuang ${ }^{1}$, Jianrong Xu ${ }^{1}$ and Yasong Du ${ }^{2^{* \dagger}}$

\begin{abstract}
Background: The aim of the current study was to investigate the utility of diffusional kurtosis imaging (DKI) in the detection of gray matter (GM) alterations in people suffering from Internet Gaming Addiction (IGA).

Methods: DKI was applied to 18 subjects with IGA and to 21 healthy controls (HC). Whole-brain voxel-based analyses were performed with the following derived parameters: mean kurtosis metrics (MK), radial kurtosis $\left(K_{\perp}\right)$, and axial kurtosis $\left(K_{/}\right)$. A significance threshold was set at $P<0.05$, AlphaSim corrected. Pearson's correlation was performed to investigate the correlations between the Chen Internet Addiction Scale (CIAS) and the DKI-derived metrics of regions that differed between groups. Additionally, we used voxel-based morphometry (VBM) to detect GM-volume differences between the two groups.
\end{abstract}

Results: Compared with the HC group, the IGA group demonstrated diffusional kurtosis parameters that were significantly less in GM of the right anterolateral cerebellum, right inferior and superior temporal gyri, right supplementary motor area, middle occipital gyrus, right precuneus, postcentral gyrus, right inferior frontal gyrus, left lateral lingual gyrus, left paracentral lobule, left anterior cingulate cortex, and median cingulate cortex. The bilateral fusiform gyrus, insula, posterior cingulate cortex (PCC), and thalamus also exhibited less diffusional kurtosis in the IGA group. MK in the left PCC and $K_{\perp}$ in the right PCC were positively correlated with CIAS scores. VBM showed that IGA subjects had higher GM volume in the right inferior and middle temporal gyri, and right parahippocampal gyrus, and lower GM volume in the left precentral gyrus.

Conclusions: The lower diffusional kurtosis parameters in IGA suggest multiple differences in brain microstructure, which may contribute to the underlying pathophysiology of IGA. DKI may provide sensitive imaging biomarkers for assessing IGA severity.

Keywords: Internet gaming addiction, Diffusional kurtosis imaging, Gray matter, Posterior cingulate cortex

\section{Introduction}

Internet use is regarded as an essential element of modern life and the number of internet users is constantly increasing. At the same time, percentages of excessive users are also on the rise [1]. One recent study, based on 24,013 fourth- to ninth-grade students recruited from 100 counties in 31 provinces in China, reported that $11.7 \%$ of the students were internet users, with a prevalence of

\footnotetext{
*Correspondence: clare1475@hotmail.com; yasongdu@163.com

${ }^{\dagger}$ Equal contributors

'Department of Radiology, Ren Ji Hospital, School of Medicine, Shanghai Jiao Tong University, Shanghai 200127, P.R. China

${ }^{2}$ Department of Child and Adolescent Psychiatry, Shanghai Mental Health Center, Shanghai Jiao Tong University, Shanghai 200030, P.R. China
}

Internet Addiction (IA) of 6.3\% [2]. IA consists of at least three subtypes: Internet Gaming Addiction (IGA), Sexual Preoccupations, and Email/Text Messaging Addiction [3], which may lead to negative consequences in daily life, such as low self-esteem, sense of loneliness, low selfdisclosure, anti-social behaviors, stronger suicidal intention, and sensation-seeking [4].

Online games are a mainstream recreation among internet users, and IGA, which broadly refers to the inability to stop playing, has become a severe social issue $[1,5]$. The etiology of IGA has yet to be studied in detail [5]. Research indicates that it is associated with a number of risk factors including certain personality traits 
(neuroticism, aggression and hostility, sensation-seeking) $[6,7]$, gaming motivations (coping with daily stressors and escapism, online relationships, mastery, control, recognition, completion, excitement, and challenge) $[8,9]$, and structural characteristics of the game (online vs. offline, positive reinforcement, the enjoyment of particular game features) [10,11]. Both structural and functional brain abnormalities associated with IGA have been reported in several studies, demonstrating that IGA could result in neuroadaptation and structural alterations as a consequence of prolonged activity in brain areas associated with addiction [12-15].

Diffusional kurtosis imaging (DKI) is an imaging technique that characterizes non-Gaussian water diffusion process [16] and quantifies the apparent diffusion coefficient and apparent diffusional kurtosis. It has been demonstrated that DKI can provide information that diffusion tensor imaging (DTI) does not, particularly regarding microstructures in the brain $[17,18]$, and thus can potentially improve the sensitivity and specificity of brain-tissue characterization in vivo. In addition, DKI permits the characterization of microstructural integrity of both gray and white matter because it is not limited to anisotropic environments [19]. The sensitivity to gray matter (GM) diffusion may be important for the examination of microstructural integrity in the brain. The aim of the present study was to investigate the application of DKI for detecting differences between the GM of an IGA population and normal controls.

\section{Method and materials \\ Theory}

Conventional DTI assumes Gaussian (i.e., unrestricted and free) diffusion. The apparent diffusivity $\left(\mathrm{D}_{\text {app }}\right)$ is derived by linearly fitting the diffusion-weighted (DW) signals acquired with one or more non-zero b-values to the following linear equation:

$$
\text { In }\left[\frac{S(\mathrm{~b})}{S(\mathrm{o})}\right]=-\mathrm{b} D_{a p p}
$$

In DKI [20,21], both apparent a diffusion coefficient $\left(D_{\text {app }}\right)$ and an apparent diffusion kurtosis $\left(K_{\text {app }}\right)$ along each applied diffusion gradient direction are estimated together by fitting the following equation to the multiple DW signals acquired using a range of b-values:

$$
\text { In }\left[\frac{S(\mathrm{~b})}{S(\mathrm{o})}\right]=-\mathrm{bD}_{a p p}+\frac{1}{6} b^{2} D_{a p p}^{2} K_{a p p},
$$

where $\mathrm{S}(\mathrm{b})$ is the DW signal intensity at a particular bvalue, and $S(0)$ the signal without applying any diffusion gradient. MK is defined as the kurtosis averaged across all directions. Note that 15 independent elements are required to construct the $4^{\text {th }}$ order diffusion kurtosis tensor (KT). KT can be transformed to a coordinate system formed by the three orthogonal eigenvectors of the $2^{\text {nd }}$ order DT $[22,23]$ :

$$
\hat{W}_{i j k l}=\sum_{i^{\prime}=1}^{3} \sum_{j^{\prime}=1}^{3} \sum_{k^{\prime}=1}^{3} \sum_{l^{\prime}=1}^{3} e_{i^{\prime} i} e_{j^{\prime} j} e_{k^{\prime} k} e_{l^{\prime} l} W_{i^{\prime} j^{\prime} k^{\prime} l^{\prime}}
$$

The kurtosis along an individual DT eigenvector can be computed from the transformed $\mathrm{KT}(\hat{W})$ :

$$
K_{i}=\frac{M D^{2}}{\lambda_{l}^{2}} \cdot \hat{W}_{i i i i .}
$$

where $\lambda_{i}$ are the eigenvalues of the DT $(\lambda 1>\lambda 2>\lambda 3)$. Axial kurtosis $\left(K_{/ /}\right)$and radial kurtosis $\left(K_{\perp}\right)$ can then be derived [22,23]:

$$
\mathrm{K}_{/ /}=K_{1}
$$

is the kurtosis along the principal DT eigenvector, and

$$
\mathrm{K}_{\perp}=\frac{K 2+K 3}{2}
$$

is the average kurtosis along the other two eigenvectors.

\section{Subject selection}

Demographic information such as gender, age, education level, and weekly Internet use were acquired through questionnaires. The present study was approved by the Ethics Committee of Ren Ji Hospital, School of Medicine, Shanghai Jiao Tong University. Full written informed consent was obtained from each participant before MRI examinations.

All subjects were interviewed by a psychiatrist regarding their medical history and underwent a basic physical examination that included blood pressure and heart rate measurements. Following the physical, subjects were assessed for psychiatric disorders using the Mini International Neuropsychiatric Interview (MINI) [24]. Exclusion criteria included a history of substance abuse or dependence, previous hospitalization for psychiatric disorders, or a history of major psychiatric disorders, such as schizophrenia, depression, anxiety disorder, or psychotic episodes.

The diagnostic questionnaire for IGA was adapted from the DSM-IV criteria for IA according to the modified Diagnostic Questionnaire for Internet Addiction criteria (the YDQ) by Beard [25]. IA falls into three subtypes, and only subjects characterized as the IGA subtype were enrolled in this study. Thus, all subjects mostly focused on online gaming when using the Internet. Eighteen subjects who satisfied these criteria were recruited from the Outpatient department of Shanghai Mental Health Center. Twenty-one age- and gender-matched healthy individuals were recruited as the control group through 
advertisements. All subjects were right-handed nonsmokers, and the IGA subjects were currently not receiving any medication or therapy for their addiction.

\section{Clinical assessments}

The Chen Internet Addiction Scale (CIAS) [26], the SelfRating Anxiety Scale (SAS) [27], the Self-rating Depression Scale (SDS) [28], and the Barratt Impulsiveness Scale-11 (BIS-11) [29] were administered to assess the participants' clinical features. All questionnaires were translated into Chinese form English for the benefit of the subjects. The cut-off scores for the CIAS, SAS, and SDS were 64, 50, and 53 respectively. The BIS-11 is a 30 -item self-report questionnaire that is widely used to measure impulsivity in a variety of populations. Higher scores indicate higher impulsivity.

\section{MRI acquisition}

All scans were acquired on a GE Signa HDxT 3.0 T MRI scanner (General Electric Medical System, Milwaukee, WI, USA) with a standard 8-channel head coil with foam padding. DWI images were acquired with three b-values $\left(0,1000\right.$, and $\left.2000 \mathrm{~s} / \mathrm{mm}^{2}\right)$ and diffusion encoding vectors along 25 nonparallel directions for each nonzero bvalue. A spin-echo echo-planar imaging sequence was applied to acquire DWI images, with the following parameters: repetition time $(\mathrm{TR})=10500 \mathrm{~ms}$, echo time $(\mathrm{TE})=$ $99.3 \mathrm{~ms}$, number of averages $=1$, slice thickness $=4 \mathrm{~mm}$, field of view $(\mathrm{FOV})=256 \mathrm{~mm} \times 256 \mathrm{~mm}$, matrix $=128 \times$ 128 , gradient length $=30.9 \mathrm{~ms}$, diffusion gradient $=39.1$ ms, and scan time $=557$ s. Signal to Noise Ratio (SNR) varied between 46 and 26 over the entire b-value range $\left(0-2000 \mathrm{~s} / \mathrm{mm}^{2}\right)$. Additionally, 3D-Fast Spoiled Gradient Recalled sequence (3D-FSPGR) images were acquired for anatomical reference with the following parameters: $\mathrm{TR}=$ $6.1 \mathrm{~ms}, \mathrm{TE}=2.8 \mathrm{~ms}$, slice thickness $=1 \mathrm{~mm}$, gap $=0$, flip angle $=15^{\circ}, \mathrm{FOV}=256 \mathrm{~mm} \times 256 \mathrm{~mm}$, matrix $=256 \times$ 256 , number of slices $=166$, and scan time $=334$ s. Axial T1 and T2W-weighted sequences were also acquired with the following parameters: T1-weighted fast-field echo sequences $(\mathrm{TR}=331 \mathrm{~ms}, \mathrm{TE}=4.6 \mathrm{~ms}, \mathrm{FOV}=256 \mathrm{~mm} \times$ $256 \mathrm{~mm}, 34$ slices, voxel size $=0.5 \mathrm{~mm} \times 0.5 \mathrm{~mm} \times 4$ $\mathrm{mm}$, and scan time $=275 \mathrm{~s}$ ); T2W turbo spin-echo sequences $(\mathrm{TR}=3013 \mathrm{~ms}, \mathrm{TE}=80 \mathrm{~ms}, \mathrm{FOV}=256 \mathrm{~mm} \times$ $256 \mathrm{~mm}$, 34 slices, voxel size $=0.5 \mathrm{~mm} \times 0.5 \mathrm{~mm} \times 4$ $\mathrm{mm}$, and scan time $=217 \mathrm{~s}$ ).

\section{Data analysis}

After confirming that the variance within each group was homogenous, two-sample t-tests were performed to determine the demographic differences between groups, and a chai-squared test was applied for gender comparison. Two-tailed p-values $\leq 0.05$ were considered statistically significant for all analyses. All statistical analyses were performed using SPSS software (v.17.0.1, IBM, USA). T1- and T2-weighted images were inspected by two experienced neuroradiologists together. No gross abnormalities were observed in either group.

For all DWI images, raw DWI data-distortion induced by eddy currents were corrected using the "eddy correct" tool in FSL (FMRIB Software Library, Oxford, UK), and non-brain tissue was removed from the image using the BET tool in FSL. Axial kurtosis $\left(K_{/ /}\right)$was derived from the diffusion tensor, while radial kurtosis $\left(K_{\perp}\right)$, and mean kurtosis (MK) were derived from the kurtosis tensor. We used the DKI processing toolbox available with Functool software version 9.4.05a (GE workstation Advantage Windows 4.4) to calculate $K_{/ /}$from the apparent diffusion, and MK and $K_{\perp}$ from the kurtosis coefficients. Voxel-based analysis (VBA) was performed using Statistical Parametric Mapping (SPM8, Wellcome Department of Imaging Neuroscience, London, UK; available at http://www.fil.ion. ucl.ac.uk/spm/software/spm8) implemented on MATLAB R2010a (MathWorks Inc., Sherborn, MA, USA). Implementation was as follows: primarily, a participant-specific b0 template was generated based on all participant data. Each b0 volume was normalized to the EPI template provided by SPM8 using a nonlinear co-registration method, with a reslicing resolution of $2 \mathrm{~mm} \times 2 \mathrm{~mm} \times 2 \mathrm{~mm}$. The normalized b0 templates were averaged and smoothed with a $6 \mathrm{~mm}$ full-width-at-half-maximum (FWHM) Gaussian kernel to yield the b0 template. Second, all original b0 images from each subject were normalized to the b0 template with a reslicing resolution of $2 \mathrm{~mm} \times 2 \mathrm{~mm} \times$ $2 \mathrm{~mm}$, and the resulting transformation matrix was applied to the $M K, K_{/ /}$, and $K_{\perp}$ maps. All acquired images were smoothed with a $6 \mathrm{~mm}$ FWHM isotropic Gaussian kernel to decrease spatial noise and to compensate for the inexact nature of normalization. In addition, a GM mask was generated from the T1-weighted (3D- FSPGR) image, which was segmented following the SPM8 segmentation routine. For each subject, the T1-weighted (3D- FSPGR) image was segmented into GM, white matter (WM) and cerebrospinal fluid, which were then normalized to the standard Montreal Neurological Institute (MNI) space using SPM8 with a reslicing resolution of $2 \mathrm{~mm} \times 2 \mathrm{~mm} \times$ $2 \mathrm{~mm}$. The normalized GM images for each subject were averaged and smoothed with a $6 \mathrm{~mm}$ FWHM Gaussian kernel, the mean of which, including all voxels with a GM probability greater than 0.5 , was converted into binary masks for further analyses.

\section{Statistical analysis}

VBA was performed for the entire brain on normalized and smoothed $\mathrm{MK}, \mathrm{K}_{/ /}$, and $\mathrm{K}_{\perp}$ maps. Two-sample $\mathrm{t}$ tests were applied to detect GM differences between groups. Correction for multiple comparisons was performed using AlphaSim software, as determined by 
Monte Carlo simulations. Statistical maps derived from the two-sample t-tests were created using a combined threshold of $P<0.001$ and a minimum cluster size of 13 voxels, yielding a corrected threshold of $P<0.05$.

\section{Correlations between GM differences in microstructure and severity of IGA}

The brain regions shown to have significantly different $\mathrm{MK}, \mathrm{K}_{\perp}$ or $\mathrm{K}_{/ /}$values in the IGA group compared with those of the control group were extracted as the region of interest (ROI) masks. These ROI masks were then projected onto the normalized and smoothed images of the 18 IGA subjects, and the $M K, K_{\perp}$, and $K_{/ /}$values were calculated for the ROIs. Next, analysis was performed to investigate the correlation between these values and CIAS scores, which measure the severity of IGA. Correlations were judged significant at $P<0.05$.

\section{Voxel-based morphometry (VBM) analysis}

Three-dimensional geometric correction was performed during reconstruction of the images. Image segmentation and registration were performed using the segmentation algorithm and the DARTEL registration algorithm incorporated in SPM8. Following the method described by van Holst [30], we used the Display function of SPM8 to manually set the image-space origin to the anterior commissure and align each image with the plane of the anterior and posterior commissures. Then, the individual native images of all participants were segmented into GM, white matter and cerebrospinal fluid (CSF). Next, GM and white matter segments were registered to a template generated from their own mean by DARTEL, before normalizing them to the Montreal Neurological Institute template space. Then, DARTEL registrations were performed with default parameter settings. Finally, we modulated the final outputs (i.e., preserving the total amount of gray matter from the original image), and smoothed the GM images with an 8-mm full-width at half-maximum Gaussian kernel. Voxel-wise comparisons of GM volume were performed between the groups using a two-sample t-test with SPM8. The significance of group differences was estimated by the theory of random Gaussian fields, and significance levels were set at uncorrected $P<0.001$ and the cluster size was set at $>100$ voxels.

\section{Results}

\section{Demographic and clinical measurements}

The demographic and behavioral measurements for the IGA and control subjects are shown in Tables 1 and 2 . No significant between-group differences in the distributions of age, gender, or education level were observed. Compared with the controls, the IGA group possessed higher CIAS, SAS, SDS, and BIS-11 scores $(P=0.000)$.

\section{Between-group analysis of DKI parameters}

Compared with the control group, the IGA group exhibited lower $\mathrm{MK}$ in the right anterior lobe of the cerebellum, left fusiform gyrus, left lingual gyrus, right inferior temporal gyrus, bilateral insula, left posterior cingulate cortex (PCC), right superior temporal gyrus, right precentral gyrus, left paracentral lobule, left anterior cingulate cortex (ACC), left median cingulate gyrus, and right supplementary motor area (SMA). Lower $\mathrm{K}_{/ /}$was observed in the right middle occipital gyrus, right anterior lobe of the cerebellum, right precuneus, right insula, bilateral thalamus, right postcentral gyrus, right $\mathrm{PCC}$, right SMA, left middle cingulate cortex (MCC), and bilateral precentral gyrus, and lower $\mathrm{K}_{\perp}$ was observed in the right inferior temporal gyrus, right orbitofrontal cortex (OFC), right fusiform gyrus, bilateral insula, left ACC, left MCC, right postcentral gyrus, left paracentral lobule, and right PCC (Tables 3, 4 and 5, Figures 1, 2, and 3) No areas showed higher $\mathrm{MK}, \mathrm{K}_{/ /}$, or $\mathrm{K}_{\perp}$.

\section{Correlations between differences in GM microstructure and IGA severity}

CIAS scores significantly and positively correlated with MK values in the left PCC and with $\mathrm{K}_{\perp}$ values in the right PCC (left PCC, MK: $\mathrm{r}=0.478, P=0.045$; right $\mathrm{PCC}, \mathrm{K}_{\perp}$ : $\mathrm{r}=0.497, P=0.036)$.

\section{VBM analysis}

VBM analysis showed that IGA subjects had higher GM volume in the right inferior and middle temporal gyri and the right parahippocampal gyrus, and lower GM volume in the left precentral gyrus compared with controls (Figure 4 and Table 6).

\section{Discussion}

As a clinically feasible extension of DTI, in addition to the orientation-dependent apparent diffusion coefficient (ADC), DKI provides additional information regarding tissue substructure [20]. DKI is more sensitive to GM, and thus provides improved GM-WM contrast [31]. Owing to the isotropic water diffusion that occurs in GM, diffusivity parameters derived from DTI have limited clinical value. DKI-derived parameters can overcome this limitation. MK is a dimensionless parameter that reflects the degree of diffusion restriction, while $K_{/ /}$and $K_{\perp}$ measure the kurtoses along the directions parallel and perpendicular to the principal diffusion direction, respectively. In contrast to conventional DTI parameters, MK is regarded as an index of tissue-microstructure complexity, such as density, orientation, degree of cell-membrane organization, axon sheaths, and myelin layers. Accordingly, parameters derived from DKI are highly sensitive to changes in microstructural tissue organization that occur during postnatal maturation of the normal brain [32,33]. Our results 
Table 1 Demographic and personality characteristics of the study participants

\begin{tabular}{lccc}
\hline & $\begin{array}{c}\text { IGA group }(\mathbf{n}=\mathbf{1 8}) \\
\text { (Mean } \pm \text { SD) }\end{array}$ & $\begin{array}{c}\text { Control group }(\mathbf{n}=\mathbf{2 1}) \\
\text { (Mean } \pm \text { SD) }\end{array}$ & p-value \\
\hline Age (years) & $20.5 \pm 3.55$ & $21.95 \pm 2.39$ & 0.138 \\
Gender (M/F) & $15 / 3$ & $18 / 3$ & 0.06 \\
Education (years) & $11.39 \pm 1.85$ & $12.38 \pm 2.13$ & 0.13 \\
Chen Internet Addiction Scale (CIAS) & $74.44 \pm 8.33$ & $38.43 \pm 9.10$ & $<0.0001$ \\
Self-Rating Anxiety Scale (SAS) & $53.66 \pm 9.71$ & $40.95 \pm 8.44$ & $<0.0001$ \\
Self-rating depression scale (SDS) & $54.72 \pm 10.44$ & $38.57 \pm 6.67$ & $<0.0001$ \\
Barratt Impulsiveness Scale-11 (BIS-11) & $63.94 \pm 8.26$ & $50.81 \pm 6.95$ & $<0.0001$ \\
\hline
\end{tabular}

Two-sample t-test was used for group comparisons. Chi-square test was used for gender comparison.

Abbreviations: IGA internet gaming addiction, SD standard deviation.

showed that the primary DKI parameters $\left(\mathrm{MK}, \mathrm{K}_{/ /}\right.$, and $K_{\perp}$ ) showed similar tendencies, but still had obvious differences depending on brain region. In GM, apart from the addition of basal dendrites and modification in tissue water content and cell packing density, it is known that changes in cortical cytoarchitecture affect how water diffuses [34,35]. MK is known to be highly sensitive in detecting general differences, but directional kurtoses are capable of providing more specific information regarding diffusion restriction along a particular direction. For instance, $K_{\perp}$ is highly sensitive to myelination [22]. More biological evidence is needed for a complete understanding of these specific observations. DTI studies have primarily reported altered WM integrity in subjects with IGA [14,36], whereas only a few studies have reported GM abnormalities in IGA subjects using VBM. IA adolescents have been demonstrated to possess lower GM volume in the left ACC, left PCC, left insula, and left lingual gyrus [15]. Furthermore, Hong et al. confirmed that adolescent boys with IA had significantly smaller cortical thicknesses in the right lateral OFC than controls, supporting the hypothesis that changes in the OFC of adolescents with IA was a neurobiological marker of addiction-related disorders in general [37]. Yuan et al. also demonstrated lower GM volume in bilateral dorsolateral prefrontal cortex (DLPFC), SMA, OFC, cerebellum, and the left rostral ACC ( $\mathrm{AACC}$ ) in adolescents with IA. GM volumes in the DLPFC, rACC, and SMA were significantly correlated with IA duration. Another study suggested that long-term IA could result in alterations in brain structure, which is likely to contribute to the chronic dysfunction observed in IA [38]. More specifically, compared with healthy subjects, IGA individuals were observed to have significant GM atrophy in the right OFC, bilateral insula and right SMA; GM volumes in the right OFC and bilateral insula were significantly positively correlated with the severity of IGA [39].

In the present study, preliminary results show a decrease in DKI parameters in the GM of IGA subjects, indicating the spread of GM damage across the brain.
GM abnormalities that were found in the OFC, insula, cingulate gyrus, SMA, ACC, PCC, precentral gyrus, cerebellum, and precuneus were consistent with previous findings $[1,15,38-40]$. Most of these areas were mentioned in a model proposed by Volkow et al. [41], who presented a model in which addiction emerges as an imbalance in information processing and integration among various brain circuits and functions. The ACC has been shown to be essential for motor control, cognition, and motivation [42], to be associated with self-control [43], and to encode the reward value during decision-making [44]. The OFC has been shown to be involved in processing emotions and cravings, in maladaptive decision-making processes, and in engaging in compulsive behaviors, each of which is integral to addiction [45]. SMA-mediated cognitive control [46] and the cingulate gyrus both contribute significantly to integrating sensory information and monitoring conflict [47]. The lingual gyrus has been linked to regulation of emotional behavior, which is a major concern in IA [15]. The precuneus possesses a critical function in processing visual spatial information and spatially guided behavior [48], and the precentral gyrus (primary motor cortex) has recently been implicated in the mechanisms underlying enhanced motivational drive for a drug [49]. Subtle modifications in the structure of various GM regions in IGA subjects may contribute to the behaviors resulting from excessive online gaming, such as issues associated with impulse control, behavioral inhibition, executive functioning, attention, and general cognitive functioning. However, as a cross-sectional study, the results of this study do not demonstrate whether the different GM microstructures found in IGA subjects preceded the development of IGA or were the consequence of gaming addiction. In addition, the changes in the parameters derived from DKI may be caused by other pathologies (such as depression or anxiety). As we listed in Tables 1 and 2, the IGA group possessed higher SAS, SDS, and BIS-11 scores than the controls, which may suggest psychiatric disorders such as depression or anxiety that could also lead to gray matter abnormalities [50,51]. It remains 
Table 2 Demographic data, CIAS, SAS, SDS, and BIS-11 scores for all participants

\begin{tabular}{|c|c|c|c|c|c|c|c|c|c|c|}
\hline \multirow[t]{3}{*}{ 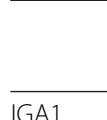 } & \multirow[t]{2}{*}{ Sex } & \multirow[t]{2}{*}{ Age } & \multirow[t]{2}{*}{ Eyr } & \multirow[t]{2}{*}{ CIAS } & \multirow[t]{2}{*}{ SAS } & \multirow[t]{2}{*}{ SDS } & \multicolumn{4}{|c|}{ BIS-11 } \\
\hline & & & & & & & Attentional key & Motor key & Non-planning key & Total score \\
\hline & M & 20 & 14 & 64 & 64 & 61 & 19 & 24 & 28 & 71 \\
\hline IGA2 & M & 14 & 12 & 65 & 56 & 54 & 18 & 22 & 26 & 66 \\
\hline IGA3 & M & 19 & 12 & 64 & 61 & 59 & 12 & 17 & 23 & 52 \\
\hline IGA4 & M & 15 & 8 & 66 & 54 & 61 & 12 & 17 & 22 & 51 \\
\hline IGA5 & $\mathrm{F}$ & 22 & 12 & 76 & 58 & 48 & 14 & 17 & 25 & 56 \\
\hline IGA6 & M & 17 & 9 & 83 & 50 & 54 & 19 & 24 & 29 & 72 \\
\hline IGA7 & M & 22 & 16 & 84 & 51 & 49 & 13 & 16 & 27 & 56 \\
\hline IGA8 & M & 18 & 10 & 75 & 46 & 61 & 15 & 18 & 31 & 64 \\
\hline IGA9 & $M$ & 23 & 11 & 87 & 56 & 68 & 23 & 24 & 29 & 76 \\
\hline IGA10 & M & 23 & 12 & 81 & 59 & 36 & 23 & 23 & 27 & 73 \\
\hline IGA11 & $M$ & 22 & 12 & 65 & 30 & 56 & 13 & 22 & 26 & 61 \\
\hline IGA12 & $M$ & 24 & 13 & 64 & 44 & 34 & 15 & 20 & 26 & 61 \\
\hline IGA13 & $M$ & 16 & 10 & 75 & 70 & 70 & 15 & 33 & 27 & 65 \\
\hline IGA14 & M & 22 & 12 & 86 & 68 & 40 & 20 & 27 & 33 & 80 \\
\hline IGA15 & $M$ & 27 & 11 & 76 & 43 & 50 & 16 & 22 & 26 & 64 \\
\hline IGA16 & F & 24 & 10 & 78 & 48 & 56 & 16 & 24 & 24 & 64 \\
\hline IGA17 & $\mathrm{F}$ & 23 & 11 & 82 & 58 & 60 & 16 & 22 & 27 & 65 \\
\hline IGA18 & $M$ & 18 & 10 & 69 & 50 & 61 & 15 & 14 & 25 & 54 \\
\hline CON1 & F & 25 & 11 & 32 & 31 & 28 & 11 & 13 & 19 & 43 \\
\hline CON2 & M & 18 & 9 & 39 & 41 & 46 & 13 & 20 & 21 & 54 \\
\hline CON3 & F & 26 & 9 & 30 & 39 & 33 & 10 & 17 & 19 & 46 \\
\hline CON4 & M & 27 & 9 & 26 & 40 & 38 & 12 & 13 & 19 & 44 \\
\hline CON5 & $M$ & 23 & 11 & 50 & 55 & 44 & 18 & 16 & 26 & 60 \\
\hline CON6 & M & 21 & 13 & 26 & 29 & 28 & 10 & 11 & 16 & 37 \\
\hline CON7 & $M$ & 21 & 13 & 52 & 36 & 35 & 13 & 18 & 25 & 56 \\
\hline CON8 & M & 22 & 14 & 32 & 34 & 39 & 10 & 15 & 19 & 44 \\
\hline CON9 & $M$ & 22 & 14 & 51 & 46 & 46 & 19 & 18 & 26 & 63 \\
\hline CON10 & $M$ & 21 & 14 & 34 & 35 & 33 & 10 & 18 & 18 & 46 \\
\hline CON11 & M & 18 & 9 & 31 & 46 & 38 & 11 & 18 & 20 & 49 \\
\hline CON12 & F & 23 & 16 & 50 & 48 & 45 & 18 & 18 & 26 & 62 \\
\hline CON13 & M & 22 & 15 & 47 & 41 & 39 & 12 & 15 & 25 & 52 \\
\hline CON14 & $M$ & 24 & 15 & 44 & 30 & 28 & 12 & 17 & 19 & 48 \\
\hline CON15 & M & 23 & 14 & 40 & 35 & 39 & 10 & 19 & 22 & 51 \\
\hline CON16 & M & 22 & 14 & 32 & 28 & 33 & 15 & 16 & 22 & 53 \\
\hline CON17 & $M$ & 22 & 12 & 42 & 46 & 44 & 14 & 16 & 22 & 52 \\
\hline CON18 & M & 19 & 12 & 28 & 43 & 34 & 10 & 17 & 15 & 42 \\
\hline CON19 & $M$ & 19 & 12 & 28 & 53 & 44 & 11 & 18 & 30 & 59 \\
\hline CON2O & M & 20 & 12 & 47 & 48 & 46 & 12 & 14 & 25 & 51 \\
\hline CON21 & $M$ & 23 & 12 & 46 & 56 & 50 & 15 & 20 & 20 & 55 \\
\hline
\end{tabular}


Table 3 Summary of MK changes between the IGA and control groups

\begin{tabular}{|c|c|c|c|c|c|c|}
\hline & \multirow[t]{2}{*}{ Peak MNI coordinate region } & \multicolumn{3}{|c|}{ Peak MNI coordinates } & \multirow[t]{2}{*}{ Number of cluster voxels } & \multirow[t]{2}{*}{ Peak $t$ value } \\
\hline & & $\mathbf{x}$ & $y$ & z & & \\
\hline 1 & Right cerebellum anterior lobe & 42 & -42 & -28 & 150 & -5.33 \\
\hline 2 & Left fusiform gyrus & -6 & -36 & 0 & 414 & -5.75 \\
\hline 3 & Left lingual gyrus & -6 & -74 & -6 & 117 & -5.12 \\
\hline 4 & Right inferior temporal gyrus & 54 & -62 & -14 & 166 & -5.25 \\
\hline 5 & Right insula & 40 & 16 & -4 & 804 & -6.95 \\
\hline 6 & Left insula & -44 & 6 & 0 & 212 & -4.95 \\
\hline 7 & Left posterior cingulate cortex & -6 & -52 & 4 & 332 & -5.30 \\
\hline 8 & Right superior temporal gyrus & 68 & -18 & 12 & 129 & -5.35 \\
\hline \multirow[t]{2}{*}{9} & Right precentral gyrus & 44 & -22 & 62 & 649 & -6.44 \\
\hline & & 50 & -8 & 32 & 82 & -4.81 \\
\hline \multirow[t]{2}{*}{10} & Left paracentral lobule & -10 & -30 & 68 & 150 & -4.83 \\
\hline & & -10 & -40 & 54 & 383 & -6.64 \\
\hline 11 & Left anterior cingulate cortex & -2 & 28 & 36 & 383 & -5.64 \\
\hline 12 & Left median cingulate gyrus & 0 & -8 & 50 & 82 & -4.27 \\
\hline 13 & Right supplementary motor area & 4 & 16 & 56 & 148 & -5.99 \\
\hline
\end{tabular}

Abbreviations: IGA internet gaming addiction, MNI Montreal Neurological Institute, MK kurtosis metrics.

unknown whether IGA is truly a unique phenomenon or simply the symptoms of underlying mental health problems [52]. Therefore, the underlying pathophysiology of the interesting findings in DKI parameters observed in GM requires further study.

Lower DKI parameters were also observed in the inferior and superior temporal gyri, middle occipital gyrus, and fusiform gyrus. The fusiform gyrus is a section of the temporal and occipital lobes that functions in processing of color information, face and body recognition, word recognition, and within-category identification, as well as the perception of emotions in facial stimuli [53-55]. The temporal regions are involved in auditory processing, comprehension, and verbal memory, whereas the occipital regions control visual processing. Recently, Han et al. [40] reported that GM volumes in the inferior temporal cortex and occipital lobe were diminished in both online game addiction and pro-gamers when compared with healthy volunteers. These two regions could be damaged by harmful visual stimuli, probably owing to the excessive exposure to visual and auditory stimulation during online games.

Table 4 Summary of differences in axial kurtosis $\left(K_{/ /}\right)$between the IGA and control groups

\begin{tabular}{|c|c|c|c|c|c|c|}
\hline & \multirow[t]{2}{*}{ Peak MNI coordinate region } & \multicolumn{3}{|c|}{ Peak MNI coordinates } & \multirow[t]{2}{*}{ Number of cluster voxels } & \multirow[t]{2}{*}{ Peak $t$ value } \\
\hline & & $\mathbf{x}$ & $y$ & $\mathbf{z}$ & & \\
\hline 1 & Right middle occipital gyrus & 50 & -68 & -16 & 130 & -4.72 \\
\hline \multirow[t]{2}{*}{2} & \multirow[t]{2}{*}{ Right cerebellum anterior lobe } & 2 & -40 & -10 & 329 & -5.03 \\
\hline & & 2 & -56 & -14 & 94 & -5.64 \\
\hline 3 & Right precuneus & 2 & -62 & 16 & 336 & -5.45 \\
\hline 4 & Right insula & 42 & -14 & 6 & 92 & -4.32 \\
\hline 5 & Right thalamus & 4 & -8 & 6 & 82 & -5.25 \\
\hline 6 & Left thalamus & -32 & -24 & 2 & 139 & -6.23 \\
\hline 7 & Right postcentral gyrus & 68 & -18 & 14 & 126 & -5.01 \\
\hline 8 & Right posterior cingulate cortex & 0 & -40 & 22 & 174 & -5.62 \\
\hline 9 & Right supplementary motor area & 4 & 16 & 54 & 309 & -5.47 \\
\hline 10 & Left median cingulate gyrus & -10 & -40 & 54 & 205 & -5.44 \\
\hline 11 & Right precentral gyrus & 36 & -24 & 68 & 342 & -5.78 \\
\hline 12 & Left precentral gyrus & -20 & -24 & 64 & 117 & -5.04 \\
\hline
\end{tabular}

Abbreviations: IGA internet gaming addiction, MNI Montreal Neurological Institute. 
Table 5 Summary of differences in radial kurtosis $\left(K_{\perp}\right)$ between the IGA and control groups

\begin{tabular}{|c|c|c|c|c|c|c|}
\hline & \multirow[t]{2}{*}{ Peak MNI coordinate region } & \multicolumn{3}{|c|}{ Peak MNI coordinates } & \multirow[t]{2}{*}{ Number of cluster voxels } & \multirow[t]{2}{*}{ Peak $t$ value } \\
\hline & & $\mathbf{x}$ & $y$ & $\mathbf{z}$ & & \\
\hline \multirow[t]{2}{*}{1} & \multirow[t]{2}{*}{ Right inferior temporal gyrus } & 44 & -42 & -28 & 129 & -5.57 \\
\hline & & 56 & -60 & -14 & 158 & -5.03 \\
\hline 2 & Right orbitofrontal cortex & 20 & 14 & -20 & 87 & -4.30 \\
\hline 3 & Right fusiform gyrus & -6 & -36 & -2 & 225 & -5.78 \\
\hline 4 & Left insula & -44 & 8 & 0 & 102 & -5.28 \\
\hline \multirow[t]{2}{*}{5} & \multirow[t]{2}{*}{ Right insula } & 40 & 16 & 2 & 288 & -6.53 \\
\hline & & 40 & -14 & 6 & 244 & -6.43 \\
\hline 6 & Left anterior cingulate cortex & -2 & 36 & 30 & 310 & -5.62 \\
\hline \multirow[t]{2}{*}{7} & \multirow[t]{2}{*}{ Left median cingulate gyrus } & -8 & -4 & 48 & 399 & -5.21 \\
\hline & & -8 & -40 & 54 & 176 & -6.18 \\
\hline 8 & Right postcentral gyrus & 38 & -30 & 50 & 307 & -5.29 \\
\hline 9 & Left paracentral lobule & -12 & -30 & 68 & 84 & -4.78 \\
\hline 10 & Right posterior cingulate cortex & 8 & -54 & 4 & 166 & -4.72 \\
\hline
\end{tabular}

Abbreviations: IGA internet gaming addiction, MNI Montreal Neurological Institute.

In the present study, the IGA group exhibited lower MK values in the left $\mathrm{PCC}$, lower $\mathrm{K}_{/ /}$values in the right $\mathrm{PCC}$, and lower $\mathrm{K}_{\perp}$ values in the right PCC. Further, positive correlations between CIAS scores and MK value were observed in left PCC and between CIAS scores and $\mathrm{K}_{\perp}$ value in right PCC. The PCC participates in visualspatial orientation and processing self-related information, is part of the default mode network [56], and often receives input via multiple sensory channels (e.g., visual, tactile, auditory, and proprioceptive). This may explain its powerful involvement in neural cue reactivity in the presence of visual and other modality stimuli. Using the reliable multisensory cue-induced activation of PCC as a biomarker may contribute to the development of successful high-tailored, subject-specific therapeutic strategies [57]. Although most previous studies have reported

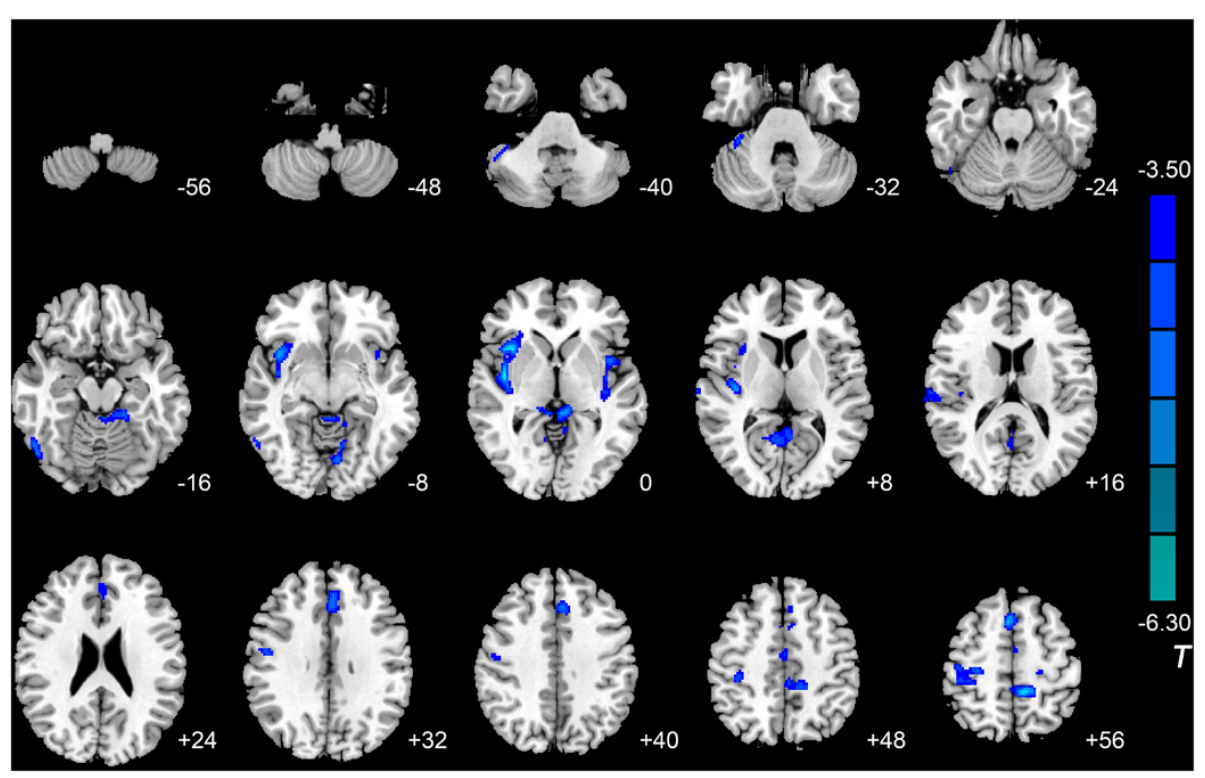

Figure 1 Significant differences in MK between IGA and HC subjects. Compared with the control group, IGA subjects exhibited lower MK in the right anterior lobe of the cerebellum, left fusiform gyrus, left lingual gyrus, right inferior temporal gyrus, bilateral insula, left posterior cingulate cortex, right superior temporal gyrus, right precentral gyrus, left paracentral lobule, left anterior cingulate gyrus, left median cingulate gyrus, and right supplementary motor area $(P<0.05$, AlphaSim-corrected). T-values are color-coded on the right. Blue indicates IGA group $<H C$. *The left section of the figure represents the patient's right side. ${ }^{*} \mathrm{HC}$, healthy control. IGA, internet gaming addiction. MK, kurtosis metrics. 


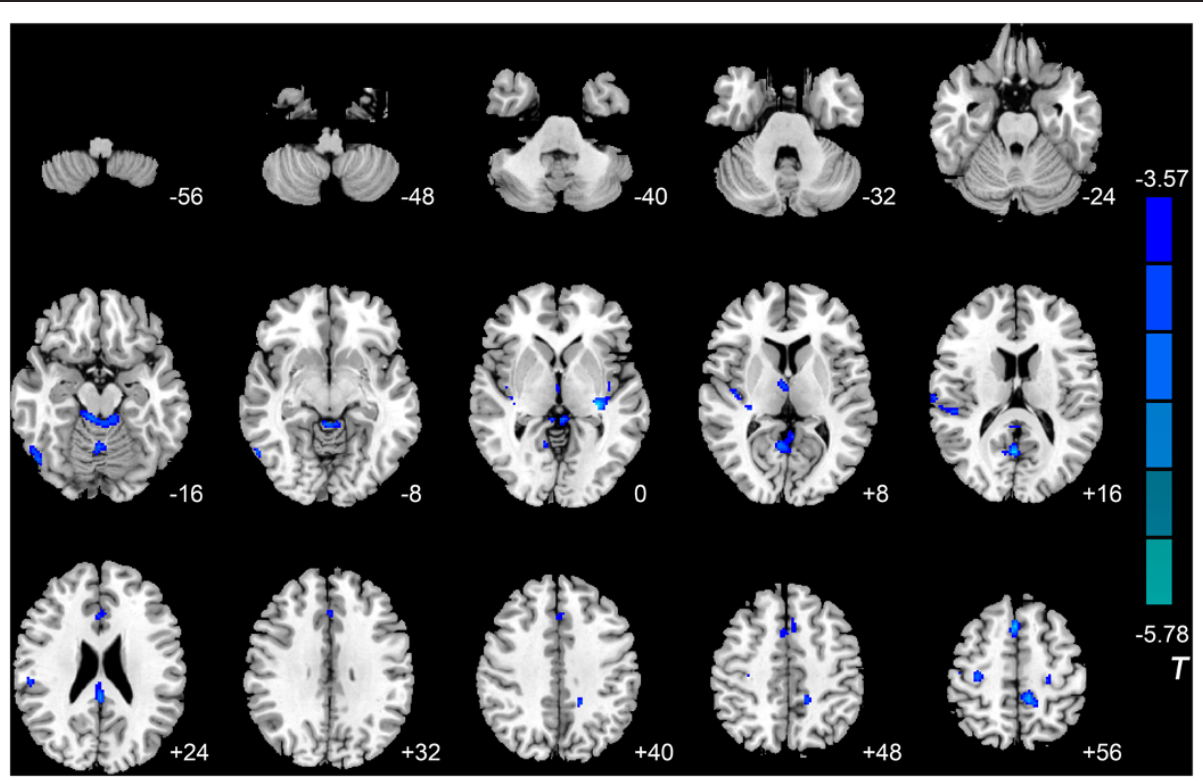

Figure 2 Significant differences in axial kurtosis $\left(\mathbf{K}_{/ /}\right)$between IGA and HC subjects. Compared with the control group, the IGA group exhibited lower $K_{/ /}$in the right middle occipital gyrus, right anterior lobe of the cerebellum, right precuneus, right insula, bilateral thalamus, right postcentral gyrus, right posterior cingulate cortex, right supplementary motor area, left median cingulate gyrus, and bilateral precentral gyrus ( $P<0.05$, AlphaSim-corrected). T-values are color-coded on the right. Blue indicates IGA group $<H C$. *The left section of the figure represents the patient's right side. ${ }^{*} \mathrm{HC}$, healthy control. IGA, internet gaming addiction.

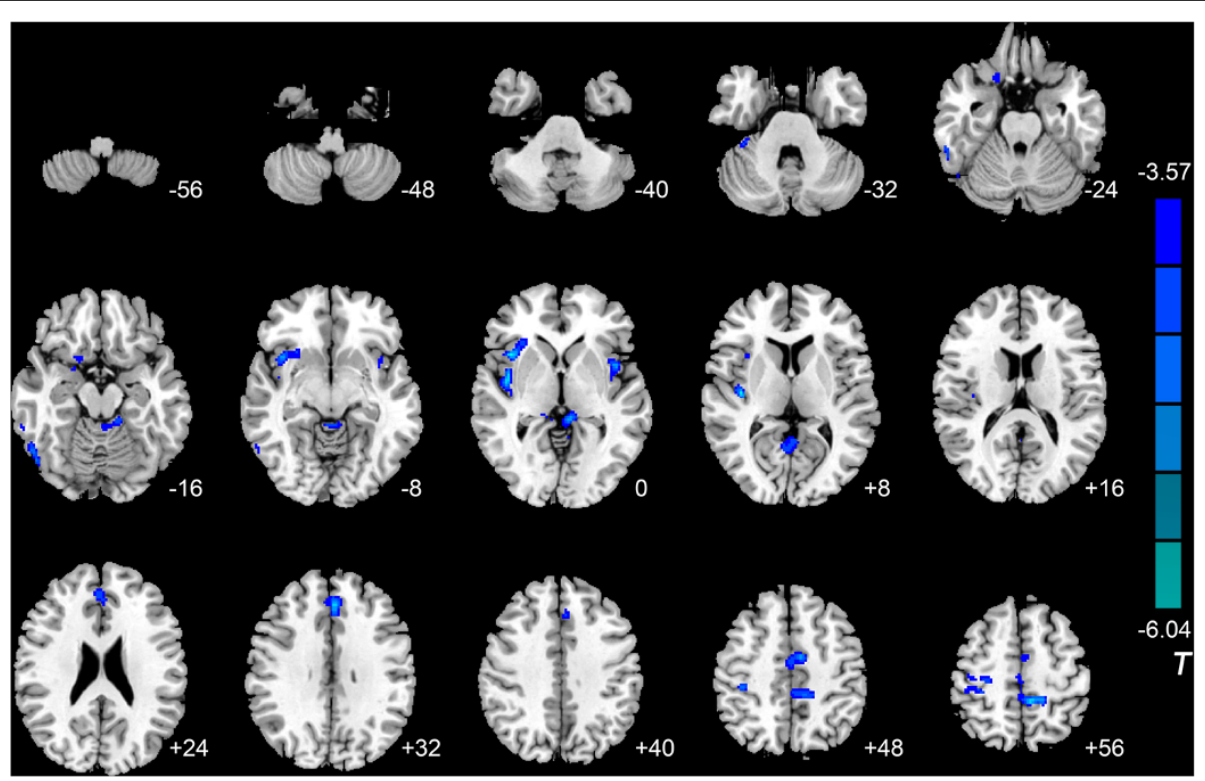

Figure 3 Significant differences in radial kurtosis $\left(\mathbf{K}_{\perp}\right)$ between IGA and HC subjects. Compared with the control group, the IGA group exhibited lower $\mathrm{K}_{\perp}$ in the right inferior temporal gyrus, right orbitofrontal cortex, right fusiform gyrus, bilateral insula, left anterior cingulate cortex, left median cingulate gyrus, right postcentral gyrus, left paracentral lobule, and right posterior cingulate cortex $(P<0.05$, AlphaSim-corrected). T-values are color-coded on the right. Blue indicates IGA group $<H C$. *The left part of the figure represents the patient's right side. *HC, healthy control. IGA, internet gaming addiction. 


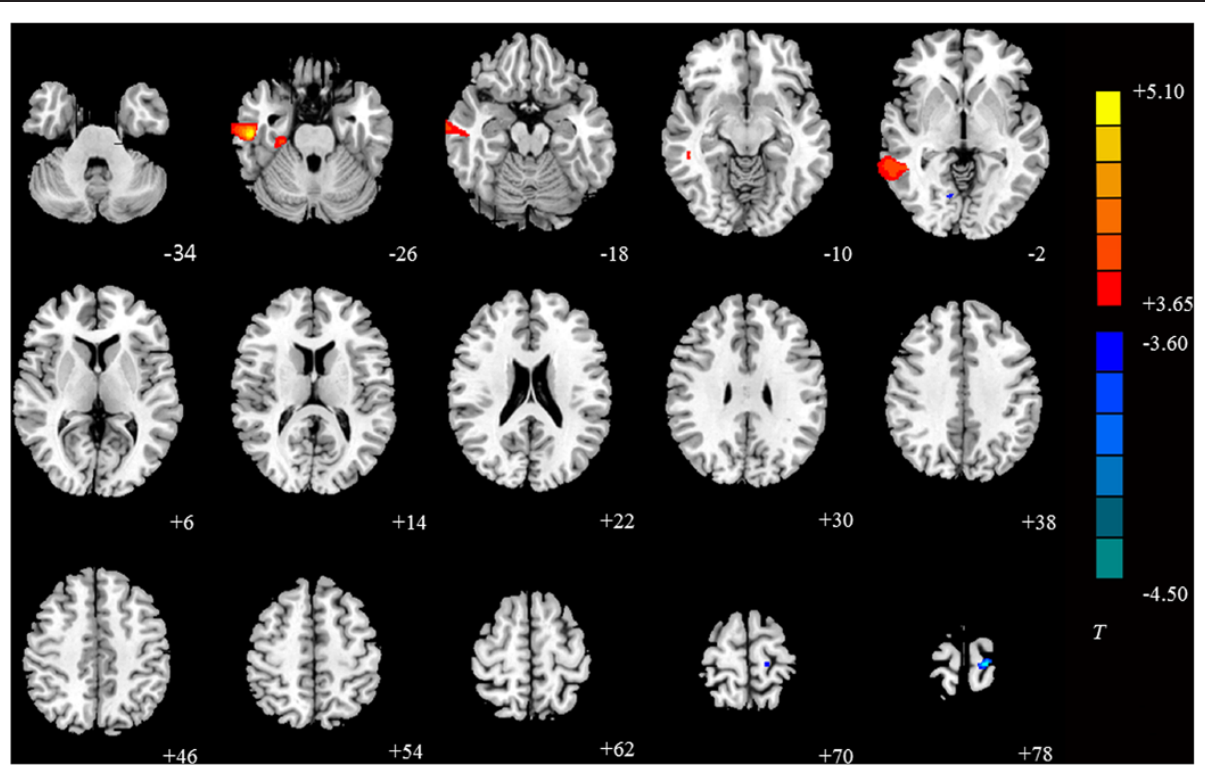

Figure 4 Significant differences in GM volume between IGA and HC subjects. The results show IGA subjects had higher GM volume in the right inferior temporal gyrus, right parahippocampal gyrus, and right middle temporal gyrus, and lower GM volume in the left precentral gyrus $(P<0.001$, cluster size was set at $>100$ voxels). T-values are color-coded on the right. Blue indicates IGA group $<H C$, yellow indicates IGA group $>$ $\mathrm{HC}$. *The left part of the figure represents the patient's right side. * HC, healthy control. IGA, internet gaming addiction.

functional and structural abnormalities in the left PCC $[15,36,58]$, we also found abnormalities in the right PCC. This inconsistency may be owing to the sensitivities of the different DKI parameters, but it still calls for further investigation of the exact underlying mechanisms.

Our VBM result was inconsistent with our result from the DKI or those from previous studies. This discrepancy may have resulted from the small sample size, the severity of our subjects' addiction, or the data-analysis methods. Additionally, the differences in the DKI parameters between IGA subjects and controls could act as a potential precursor of further modifications in the gray matter not yet expressed in the VBM-based analysis. Further studies are required to clarify this pointe.

There are several potential limitations of the present study. First, the relatively small sample size may limit the generalization of the results; more biological evidence is warranted to understand these specific observations. Second, because the study focused on only one subtype of IA, the results may not extend to the other IA subtypes. Third, a GM mask was applied for the analysis using segmented tissue maps from high-resolution 3D-SPGR images. The results of the mask depended on the segmentation results, and further methodological advances might improve the current correction technique. Last, potential confounding factors such as levels of physical activity and school performance were not excluded. Future prospective studies should therefore focus on clarifying the causal relationship between IGA and psychological measures.

\section{Conclusion}

DKI can detect subtle differences in GM microstructure between IGA and $\mathrm{HC}$ individuals. The decrease of $\mathrm{K}_{\perp}$, $\mathrm{K}_{/ /}$, and MK values in IGA subjects indicated multiple changes in brain microstructure, which may be implicated in the underlying pathophysiology of IGA. Furthermore, the DKI model can provide sensitive imaging biomarkers for assessing the severity of IGA.

Table 6 Summary of GM volume changes in the IGA group compared with the control group

\begin{tabular}{|c|c|c|c|c|c|c|}
\hline & \multirow[t]{2}{*}{ Peak MNI coordinate region } & \multicolumn{3}{|c|}{ Peak MNI coordinates } & \multirow[t]{2}{*}{ Number of cluster voxels } & \multirow[t]{2}{*}{ Peak T value } \\
\hline & & $\mathbf{x}$ & $\mathbf{y}$ & $\mathbf{z}$ & & \\
\hline 1 & Right inferior temporal gyrus & 54 & -19 & -27 & 1012 & 5.39 \\
\hline 2 & Right parahippocampal & 27 & -24 & -24 & 198 & 4.07 \\
\hline 3 & Right middle temporal gyrus & 48 & -45 & -3 & 837 & 4.35 \\
\hline 4 & Left precentral gyrus & -18 & -28 & 76 & 190 & -4.71 \\
\hline
\end{tabular}

Abbreviations: IGA internet gaming addiction, MNI Montreal Neurological Institute, GM gray matter. 


\section{Competing interests}

The authors declare that they have no competing interests.

\section{Authors' contributions}

$Y Z, J S, J X$, and $Y D$ conceived and designed the experiments. $Y S, X C$, and $Z Z$ performed the experiments. Authors $Y Z, Y S$, and WD analyzed the data. YS and $Y Z$ wrote the paper. All authors read and approved the final manuscript.

\section{Acknowledgments}

This research was supported by grants from the National Natural Science Foundation of China (No. 81171325), Shanghai Science and Technology Committee Medical Guide Project (No. 114119a0900), National Natural Science Foundation of China (No. 81201172), National Natural Science Foundation of China (No. 81371622), and Shanghai Leading Academic Discipline Project (Project No. S30203). The funders played no further role in the study design, data collection and analysis, decision to publish, or preparation of the manuscript.

The authors thank Dr. Zhenyu Zhou and Yong Zhang of GE Healthcare for their technical support.

Received: 23 April 2014 Accepted: 1 October 2014

Published: 24 October 2014

\section{References}

1. Kuss DJ, Griffiths MD: Internet and gaming addiction: a systematic literature review of neuroimaging studies. Brain Sci 2012, 2:347-374.

2. Li YZX, Lu F, Zhang Q, Wang Y: Internet addiction among elementary and middle school students in china: a nationally representative sample study. Cyberpsychol Behav Soc Netw 2014, 7:111-116.

3. Block JJ: Prevalence underestimated in problematic Internet use study. CNS Spectr 2007, 12:14. author reply 15.

4. Heo J, Oh J, Subramanian SV, Kim Y, Kawachi I: Addictive internet use among Korean adolescents: A National Survey. PLoS One 2014, 9:e87819. doi:87810.81371/journal.pone.0087819.

5. Kuss DJ: Internet gaming addiction: current perspectives. Psychol Res Behav Manag 2013, 6:125-137.

6. Mehroof M, Griffiths MD: Online gaming addiction: the role of sensation seeking, self-control, neuroticism, aggression, state anxiety, and trait anxiety. Cyberpsychol Behav Soc Netw 2010, 13:313-316.

7. Chiu SI, Lee JZ, Huang DH: Video game addiction in children and teenagers in Taiwan. Cyberpsychol Behav 2004, 7:571-581.

8. $\mathrm{Ng} \mathrm{BD}$, Wiemer-Hastings P: Addiction to the internet and online gaming. Cyberpsychol Behav 2005, 8:110-113.

9. Daniel LKPH, Mark DG: The role of structural characteristics in problematic video game play: an empirical study. Int J Ment Health Addict 2011, 9:320-333.

10. Chumbley J, Griffiths M: Affect and the computer game player: the effect of gender, personality, and game reinforcement structure on affective responses to computer game-play. Cyberpsychol Behav 2006, 9:308-316.

11. King D, Delfabbro P, Griffiths M: The convergence of gambling and digital media: implications for gambling in young people. J Gamb/ Stud 2010, 26:175-187.

12. Feng $Q$, Chen X, Sun J, Zhou Y, Sun Y, Ding W, Zhang Y, Zhuang Z, Xu J, Du $Y$ : Voxel-level comparison of arterial spin-labeled perfusion magnetic resonance imaging in adolescents with internet gaming addiction. Behav Brain Funct 2013, 9:33.

13. Ding WN, Sun JH, Sun YW, Zhou Y, Li L, Xu JR, Du YS: Altered default network resting-state functional connectivity in adolescents with Internet gaming addiction. PLOS One 2013, 8:e59902.

14. Lin F, Zhou Y, Du Y, Qin L, Zhao Z, Xu J, Lei H: Abnormal white matter integrity in adolescents with internet addiction disorder: a tract-based spatial statistics study. PLoS One 2012, 7:e30253.

15. Zhou Y, Lin FC, Du YS, Qin LD, Zhao ZM, Xu JR, Lei H: Gray matter abnormalities in Internet addiction: a voxel-based morphometry study. Eur J Radiol 2011, 79:92-95.

16. Jensen $\mathrm{JH}$, Helpern JA: MRI quantification of non-Gaussian water diffusion by kurtosis analysis. NMR Biomed 2010, 23:698-710.

17. Jensen JH, Falangola MF, Hu C, Tabesh A, Rapalino O, Lo C, Helpern JA: Preliminary observations of increased diffusional kurtosis in human brain following recent cerebral infarction. NMR Biomed 2011, 24:452-457.
18. Raab P, Hattingen E, Franz K, Zanella FE, Lanfermann H: Cerebral gliomas: diffusional kurtosis imaging analysis of microstructural differences. Radiology 2010, 254:876-881.

19. Lu HJJ, Ramani A, Helpern JA: Three-dimensional characterization of non-gaussian water diffusion in humans using diffusion kurtosis imaging. NMR Biomed 2006, 19:236-247.

20. Jensen JH, Helpern JA, Ramani A, Lu H, Kaczynski K: Diffusional kurtosis imaging: the quantification of non-gaussian water diffusion by means of magnetic resonance imaging. Magn Reson Med 2005, 53:1432-1440.

21. Liu CBR, Acar B, Moseley ME: Characterizing non-Gaussian diffusion by using generalized diffusion tensors. Magn Reson Med 2004, 51:924-937.

22. Hui ESCM, Qi L, Wu EX: Towards better MR characterization of neural tissues using directional diffusion kurtosis analysis. Neuroimage 2008, 42:122-134.

23. Qi L, WE WY: D-eigenvalues of diffusion kurtosis tensors. J Comput Appl Math 2008, 221:150-157.

24. Lecrubier YSD, Weiller E, Amorim P, Bonora I, Harnett Sheehan K, Janavs J, Dunbar GC: The Mini International Neuropsychiatric Interview (MINI). A short diagnostic structured interview: reliability and validity according to the CIDI. Eur Psychiatry 1997, 12:224-231.

25. Beard KW, Wolf EM: Modification in the proposed diagnostic criteria for Internet addiction. Cyberpsychol Behav 2001, 4:377-383.

26. Chen SHWL, Su YJ, Wu HM, Yang PF: Development of Chinese Internet addcition scale and its psychometric study. Chinese J Psychol 2003, 45:279-294.

27. Zung WW: A rating instrument for anxiety disorders. Psychosomatics 1971, 12:371-379.

28. Zung WW: A self-rating depression scale. Arch Gen Psychiatry 1965, 12:63-70.

29. Patton JH, Stanford MS, Barratt ES: Factor structure of the Barratt impulsiveness scale. J Clin Psychol 1995, 51:768-774.

30. van Holst RJ, de Ruiter MB, van den Brink W, Veltman DJ, Goudriaan AE: $A$ voxel-based morphometry study comparing problem gamblers, alcohol abusers, and healthy controls. Drug Alcohol Depend 2012, 124:142-148.

31. Feindel KW: Can we develop pathology-specific MRI contrast for "MR-negative" epilepsy? Epilepsia 2013, 54(Suppl 2):71-74.

32. Latt J, Nilsson M, Wirestam R, Stahlberg F, Karlsson N, Johansson M, Sundgren $\mathrm{PC}$, van Westen D: Regional values of diffusional kurtosis estimates in the healthy brain. J Magn Reson Imaging 2013, 37:610-618.

33. Cheung MMHE, Chan KC, Helpern JA, Qi L, Wu EX: Does diffusion kurtosis imaging lead to better neural tissue characterization? A rodent brain maturation study. Neuroimage 2009, 45:386-392.

34. Bockhorst KH, Narayana PA, Liu R, Ahobila-Vijjula P, Ramu J, Kamel M, Wosik J, Bockhorst T, Hahn K, Hasan KM, Perez-Polo JR: Early postnatal development of rat brain: in vivo diffusion tensor imaging. J Neurosci Res 2008, 86:1520-1528.

35. Sizonenko SV, Camm EJ, Garbow JR, Maier SE, Inder TE, Williams CE, Neil Jر, Huppi PS: Developmental changes and injury induced disruption of the radial organization of the cortex in the immature rat brain revealed by in vivo diffusion tensor MRI. Cereb Cortex 2007, 17:2609-2617.

36. Dong G, DeVito E, Huang J, Du X: Diffusion tensor imaging reveals thalamus and posterior cingulate cortex abnormalities in internet gaming addicts. J Psychiatr Res 2012, 46:1212-1216.

37. Hong SB, Kim JW, Choi EJ, Kim HH, Suh JE, Kim CD, Klauser P, Whittle S, Yucel M, Pantelis C, Yi SH: Reduced orbitofrontal cortical thickness in male adolescents with internet addiction. Behav Brain Funct 2013, 9:11.

38. Yuan K, Qin W, Wang G, Zeng F, Zhao L, Yang X, Liu P, Liu J, Sun J, von Deneen KM, Gong Q, Liu Y, Tian J: Microstructure abnormalities in adolescents with internet addiction disorder. PLOS One 2011, 6:e20708.

39. Weng CB, Qian RB, Fu XM, Lin B, Han XP, Niu CS, Wang YH: Gray matter and white matter abnormalities in online game addiction. Eur J Radiol 2013, 82:1308-1312.

40. Han DH, Lyoo IK, Renshaw PF: Differential regional gray matter volumes in patients with on-line game addiction and professional gamers. J Psychiatr Res 2012, 46:507-515.

41. Volkow ND, Wang GJ, Fowler JS, Tomasi D, Telang F, Baler R: Addiction: decreased reward sensitivity and increased expectation sensitivity conspire to overwhelm the brain's control circuit. Bioessays 2010, 32:748-755

42. Goldstein RZ, Alia-Klein N, Tomasi D, Carrillo JH, Maloney T, Woicik PA, Wang R, Telang F, Volkow ND: Anterior cingulate cortex hypoactivations to 
an emotionally salient task in cocaine addiction. Proc Natl Acad Sci U S A 2009, 106:9453-9458.

43. Ko CH, Liu GC, Hsiao S, Yen JY, Yang MJ, Lin WC, Yen CF, Chen CS: Brain activities associated with gaming urge of online gaming addiction. J Psychiatr Res 2009, 43:739-747.

44. Wallis JD, Kennerley SW: Contrasting reward signals in the orbitofrontal cortex and anterior cingulate cortex. Ann N Y Acad Sci 2011, 1239:33-42.

45. Schoenbaum G, Roesch MR, Stalnaker TA: Orbitofrontal cortex, decisionmaking and drug addiction. Trends Neurosci 2006, 29:116-124.

46. Li CS, Sinha R: Inhibitory control and emotional stress regulation: neuroimaging evidence for frontal-limbic dysfunction in psycho-stimulant addiction. Neurosci Biobehav Rev 2008, 32:581-597.

47. Liu J, Gao XP, Osunde I, Li X, Zhou SK, Zheng HR, Li LJ: Increased regional homogeneity in internet addiction disorder: a resting state functional magnetic resonance imaging study. Chin Med J (Engl) 2010, 123:1904-1908.

48. Cavanna AE, Trimble MR: The precuneus: a review of its functional anatomy and behavioural correlates. Brain 2006, 129:564-583.

49. Volkow ND, Wang GJ, Fowler JS, Tomasi D, Telang F: Addiction: beyond dopamine reward circuitry. Proc Natl Acad Sci U S A 2011, 108:15037-15042

50. Grieve SM, Korgaonkar MS, Koslow SH, Gordon E, Williams LM: Widespread reductions in gray matter volume in depression. Neuroimage Clin 2013, 3:332-339.

51. Shang J, Fu Y, Ren Z, Zhang T, Du M, Gong Q, Lui S, Zhang W: The common traits of the ACC and PFC in anxiety disorders in the DSM-5: meta-analysis of voxel-based morphometry studies. PLOS One 2014 9:e93432.

52. Ferguson CJ, Coulson M, Barnett J: A meta-analysis of pathological gaming prevalence and comorbidity with mental health, academic and social problems. J Psychiatr Res 2011, 45:1573-1578.

53. Parvizi J, Jacques C, Foster BL, Witthoft N, Rangarajan $V$, Weiner KS, Grill-Spector K: Electrical stimulation of human fusiform face-selective regions distorts face perception. J Neurosci 2012, 32:14915-14920.

54. Dehaene $S$, Cohen $L$ : The unique role of the visual word form area in reading. Trends Cogn Sci 2011, 15:254-262.

55. Weiner KSGG, Caspers J, Chuapoco MR, Mohlberg H, Zilles K, Amunts K, Grill-Spector K: The mid-fusiform sulcus: a landmark identifying both cytoarchitectonic and functional divisions of human ventral temporal cortex. Neuroimage 2014, 1:453-465.

56. Andrews-Hanna JR, Reidler JS, Sepulcre J, Poulin R, Buckner RL: Functional-anatomic fractionation of the brain's default network. Neuron 2010, 65:550-562.

57. Yalachkov $Y$, Kaiser J, Naumer MJ: Functional neuroimaging studies in addiction: multisensory drug stimuli and neural cue reactivity. Neurosci Biobehav Rev 2012, 36:825-835.

58. Ko CH, Liu GC, Yen JY, Chen CY, Yen CF, Chen CS: Brain correlates of craving for online gaming under cue exposure in subjects with Internet gaming addiction and in remitted subjects. Addict Biol 2013, 18:559-569.

doi:10.1186/1744-9081-10-37

Cite this article as: Sun et al: Assessment of in vivo microstructure alterations in gray matter using DKI in internet gaming addiction. Behavioral and Brain Functions 2014 10:37.

\section{Submit your next manuscript to BioMed Central and take full advantage of:}

- Convenient online submission

- Thorough peer review

- No space constraints or color figure charges

- Immediate publication on acceptance

- Inclusion in PubMed, CAS, Scopus and Google Scholar

- Research which is freely available for redistribution

Submit your manuscript at www.biomedcentral.com/submit
Ciomed Central 